\title{
高压油洜凸轮轴激光增材制造梯度耐磨层研究
}

\author{
石 岩 李云峰 刘 佳 袁振玉 \\ (长春理工大学机电工程学院 长春 130022)
}

摘要 : 为改善高压油洜凸轮轴的耐磨性能与工作可靠性, 采用 $5 \mathrm{~kW} \mathrm{CO}_{2}$ 激光器、四轴联动数控机床及载气式同轴送粉系统 等设备对复杂形貌高压油泉凸轮轴进行激光增材制造梯度耐磨涂层工艺研究。通过梯度耐磨涂层结构设计、凸轮边缘防塌陷 工装研制与凸轮运动轨迹控制等关键技术的研究, 实现了均匀无缺陷且凸轮边缘保护良好的高硬度梯度耐磨涂层的制备。同 时, 利用扫描电镜、 $X$ 射线衍射仪、显微硬度计及光学显微镜等分析、测试仪器对梯度耐磨涂层宏观形貌与微观组织进行表 征。利用高压喷油洜试验台对激光增材制造与渗碳淬火处理凸轮轴进行使用寿命对比考核测试。结果表明, 梯度涂层中主要 由 $\gamma-\mathrm{Co}$ 固溶体、 $\mathrm{Co}_{3} \mathrm{~B}$ 及 $\mathrm{M}_{23} \mathrm{C}_{6}$ 等相组成 ; 其中过渡层与耐磨层还存在 $\mathrm{WC}$ 与 $\mathrm{W}_{2} \mathrm{C}$ 形式的增强相，微观组织主要表现为灰 白色共晶组织与深灰色枝晶组织; 梯度涂层与基体形成良好的冶金结合 ; 增材处理后的凸轮表面耐磨损性能明显提高，使用 寿命较渗碳淬火凸轮轴提高约 $65 \%$ 。

关键词：激光增材制造；凸轮轴；梯度涂层 ; 微观组织; 耐磨性能

中图分类号 : TG156

\section{Research of Gradient Wear-resisting Coating Produced by Laser Additive Manufacturing on High-pressure Pump Camshaft}

\author{
SHI Yan LI Yunfeng LIU Jia YUAN Zhenyu \\ (School of Electromechanical Engineering,
}

Changchun University of Science and Technology, Changchun 130022)

\begin{abstract}
To improve the wear resistance and operational reliability of high-pressure pump camshaft. The research of gradient wear-resisting coating produced by laser additive manufacturing on complex morphology high-pressure pump camshaft using the 5 $\mathrm{kW} \mathrm{CO}$ laser, four-axis CNC machines and the co-axial powder feeding system. Based on the research of the gradient coating, the edge anti-collapse fixture and the movement control of cam, and realized the preparation of uniform high-hardness gradient coating without defects and the good protection of cam's edges. The macromorphology and microstructures are characterized by using laser microscope, SEM, XRD and microhardness tester. Comparison tests of service lives of the additive manufacturing camshafts and the cemented quenching camshafts are carried out on the high pressure pump test-bed. The results show that the primary phases of gradient coating are $\gamma$-Co solid solution, $\mathrm{Co}_{3} \mathrm{~B}, \mathrm{M}_{23} \mathrm{C}_{6}$ and etc. The reinforcing phases are $\mathrm{WC}$ and $\mathrm{W}_{2} \mathrm{C}$ in transition layers and wear-resisting layer. The microstructures of gradient coating contains primary dendrites and interdendritic eutectics. The metallurgical bonding forms well between the gradient coating and the cam. Compared with the cemented quenching camshafts, the useful life of the additive manufacturing camshafts is increased by $65 \%$.
\end{abstract}

Key words : laser additive manufacturing ; camshaft ; gradient coating ; microstructure ; wear resistance

\section{0 前言}

凸轮轴广泛应用于柴油发动机, 由于节能减排 的需要, 新型发动机的重量在逐渐减轻, 体积逐渐 减小, 凸轮轴所承受载荷逐渐增大, 如常规车用凸 轮轴载荷一般为 $100 \sim 120 \mathrm{MPa}$, 但对于新型高功率 密度柴油机的凸轮轴载荷会达到 $180 \mathrm{MPa}$ ，常规的 渗碳淬火工艺已无法满足其使用需要, 短周期内会

*国家装备预研基金资助项目(51318060104)。20160419 收到初稿， 20160808 收到修改稿
出现粘着磨损、疲劳点蚀与表面剥落等失效形式, 从而影响发动机的使用寿命。因此, 大幅度提高凸 轮轴凸轮表面耐磨性能是迫切需要解决的问题。

为提高凸轮轴的使用寿命，已有部分研究人员 针对其表面强化方法进行了研究。如王大承等 ${ }^{[1-2]}$ 对凸轮轴进行了激光相变硬化与激光熔凝处理的研 究。卢求元等 ${ }^{[3]}$ 在发动机凸轮表面分别制备类金刚 石和 CrTiAlN 两种耐磨减摩薄膜。虽然以上研究对 凸轮轴凸轮表面性能都实现了不同程度的改善，但 受到材料本身性能与镀膜厚度的限制，较难实现凸 轮表面性能的大幅提高。采用激光增材制造技术可 
在零件表面熔融堆积性能优良的金属材料，使零部 件表面性能得到显著增强 ${ }^{[4-10]}$ 。同时，由于凸轮表 面属于复杂回转体曲面，因此在实现凸轮运动轨迹 控制与涂层裂纹消除以及涂层厚度均匀性等方面存 在难题, 并且在增材过程中凸轮边缘可能出现塌陷 现象, 对后续精加工带来不利影响。为此, 沈斌 等 ${ }^{[1-12]}$ 研究了凸轮激光熔覆的轨迹控制及熔覆层厚 度的可控性与均匀性。虽然解决了凸轮熔覆层均匀 性的问题, 但涂层厚度只有 $1 \mathrm{~mm}$ 且凸轮轮廓复杂 程度不高。综上所述, 针对凸轮表面激光熔覆处理 的研究主要集中在低厚度涂层的制备。有关凸轮表 面激光增材制备高厚度耐磨涂层的研究较少。

论文以载荷 $180 \mathrm{MPa}$ 高压油原凸轮轴为研究对 象，研究在复杂结构凸轮表面增材制造梯度耐磨层， 提高凸轮轴耐磨性能与工作可靠性。在实际应用中 具有较大的经济意义和实用价值, 对其他复杂曲面 零件表面增材加工具有参考价值。

\section{1 试验材料及方法}

\section{1 试验材料}

凸轮轴材料为 $40 \mathrm{CrMnTi}$, 调质处理, 硬度为 $35 \mathrm{HRC}$, 如图 1 所示, 两个互呈 $60^{\circ}$ 错开的三角形 凸轮为待处理部位。凸轮桃部尺寸单边减小 $2.5 \mathrm{~mm}$ 加工, 其余部位按原工艺预留 $0.2 \mathrm{~mm}$ 余量。试验 用 Co50 合金粉末, 粒度为 $45 \sim 109 \mu \mathrm{m}$, 熔点 1100 ${ }^{\circ} \mathrm{C}$, 其化学成分质量分数为 $w_{\mathrm{Cr}}=21.6 \%, w_{\mathrm{Ni}}=10.4 \%$, $w_{\mathrm{Si}}=1.8 \%, w_{\mathrm{B}}=2.55 \%, w_{\mathrm{C}}=0.1 \%, w_{\mathrm{W}}=5.62 \%$, Co 余量。 $\mathrm{Ni} / \mathrm{WC}$ 粉末粒度为 $45 \sim 109 \mu \mathrm{m}$ 。其化学成分 质量分数为: $w_{\mathrm{C}}=1.5 \% \sim 3.0 \%, w_{\mathrm{B}}=1.5 \% \sim 3.5 \%$, $w_{\mathrm{Si}}=1.0 \% \sim 4.0 \%, w_{\mathrm{Fe}}<14 \%, w_{\mathrm{Cr}}=8.0 \% \sim 12.0 \%$, $w_{\mathrm{W}}=5.62 \%, \mathrm{Ni}$ 余量。稀土选用纯度为 $99.9 \%$ 的 $\mathrm{CeO}_{2}$ 粉末。

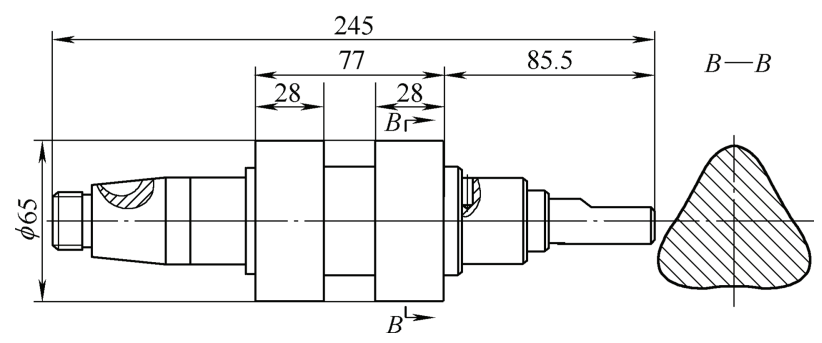

图 1 凸轮轴外形结构尺寸

\section{2 激光增材制造装置及增材过程}

增材制造试验在 SLC-X15×30 型四轴联动数控 激光加工机上进行, 凸轮轴装夹在转台上以实现旋 转运动，工作台实现 $X-Y-Z$ 三轴移动，如图 2 所示。 激光发生器采用德国 RofinDC050 型 $\mathrm{CO}_{2}$ 激光器，
波长 $10.6 \mu \mathrm{m}$, 光束模式为 TEM00, 光束质量因数 $k \geqslant 0.9$, 激光束经反射聚焦后, 离焦照射在凸轮表面, 光斑直径 $4 \mathrm{~mm}$ 。采用同轴送粉头实现与激光同轴 送粉方式完成增材制造过程。选用两台载气式送粉 器为同轴送粉头供粉, 送粉转速 $0.5 \sim 0.9 \mathrm{r} / \mathrm{s}$, 载粉 气体为氩气, 送粉气体流量为 $700 \mathrm{~L} / \mathrm{h}$, 同轴送粉头 通氩气作为保护气体，气体流量为 $14 \mathrm{~L} / \mathrm{min}^{\circ}$ 。增材 制造试验前采用 400\#砂纸对凸轮表面进行毛化处 理并用丙酮清洗。为减小增材过程中的开裂倾向， 试验开始前将凸轮轴在加热炉中 $300 \sim 350{ }^{\circ} \mathrm{C}$ 保温 $1 \mathrm{~h}$ 。激光增材制造后将凸轮轴再次放入 $300 \sim 350$ ${ }^{\circ} \mathrm{C}$ 的加热炉中保温 $2 \mathrm{~h}$ 后随炉冷却至室温。

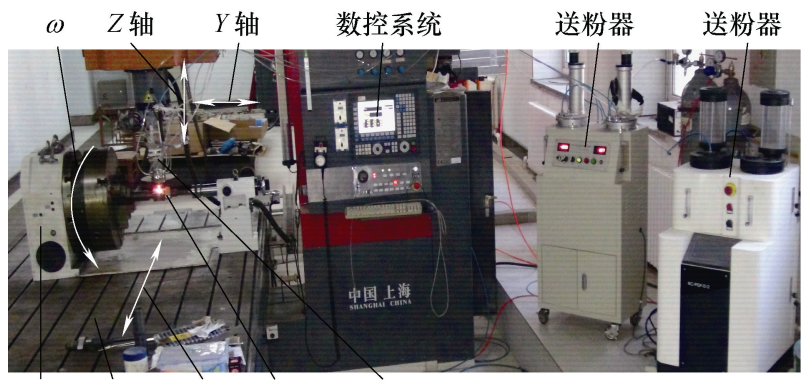

转台 工作台 $X$ 轴凸轮轴 同轴送粉头

图 2 凸轮轴激光增材制造设备实物图

\section{3 激光增材分析试样的制备及测试方法}

将激光增材制造后的凸轮轴经切割、镶嵌、研 磨、抛光与腐蚀后制成微观分析试样。腐蚀液采用 体积比为 3: 1 的 $\mathrm{HCl}$ 与 $\mathrm{HNO}_{3}$ 溶液。利用 ME61 光学显微镜拍摄梯度涂层横截面形貌。在 JSM-6510F 型扫描电镜下拍摄梯度涂层的微观组织 形貌，其加速电压为 $20 \mathrm{kV}$ 。采用 XRD-6000 型 X 射线衍射仪进行物相分析，选用铜靶和石墨滤波片， 工作电压为 $50 \mathrm{kV}$, 工作电流为 $300 \mathrm{~mA}$ 。显微硬度 测试在 MH-60 显微硬度测量仪上进行, 载荷质量 $200 \mathrm{~g} ，$ 加载时间 $10 \mathrm{~s}$ 。

\section{4 凸轮耐磨性能分析设备及测试方法}

采用德国 BOSCH EPS $30 \mathrm{~kW}$ 高压喷油洜试验 台与法国 EFS 高压共轨控制系统对激光增材制造与 渗碳淬火凸轮轴进行使用寿命对比考核测试。测试 以 $10 \mathrm{~h}$ 为试验周期, 停试验台 $20 \mathrm{~min}$, 检验凸轮磨 损情况, 若凸轮出现磨损, 则立即停止试验, 并进 行检测与判断。测试依据 GB/T25365.1-2010 柴油机 电控共轨喷油系统总成与 GB/T25368-2010 柴油机 电控共轨喷油系统高压供油厡总成。

\section{2 激光增材梯度涂层设计}

激光增材制造高厚度、高硬度耐磨涂层极易产 
生裂纹及缺陷 ${ }^{[13-16]}$ 。为此, 通过采用材料成分渐变 的梯度涂层结构，来实现凸轮表面增材制造无裂纹 与缺陷的梯度耐磨层。由于 Co50 合金粉末具有较 高的硬度与耐磨性能且韧性与润湿性较好, 故梯度 耐磨涂层采用 Co50 为主体成分, 通过在 Co50 中添 加不同含量的稀土与 WC 颗粒增强相来实现梯度过 渡。梯度层设计为一层 $0.4 \mathrm{~mm}$ 的连接层、两层各 $0.8 \mathrm{~mm}$ 的过渡层及最外一层 $1 \mathrm{~mm}$ 的耐磨层结构 (图 3)。连接层起到实现增材制造层与基体有效连接 的作用，该层直接采用 Co50 选用 $30 \%$ 的大稀释率 来实现。这样既可保证增材制造层与基体形成有效 牢固的冶金结合, 又因较大的稀释率可使连接层与 基体的热胀系数趋于接近, 减小开裂倾向。前期研 究表明, 稀土质量分数在 $0.1 \%$ 左右时, 可细化晶粒 并控制涂层无裂纹产生 ${ }^{[17]}$ 。考虑 $30 \%$ 稀释率的影 响, 连接层添加 $0.14 \%$ 的稀土元素, 进一步提高其 韧性; 两层过渡层分别采用 $\mathrm{Co} 50+0.1 \% \mathrm{CeO}_{2}+3 \% \mathrm{Ni} /$ $\mathrm{WC}$ 与 $\mathrm{Co} 50+0.1 \% \mathrm{CeO}_{2}+5 \% \mathrm{Ni} / \mathrm{WC}$ 两种成分结构， 过渡层 $\mathrm{b}$ 与过渡层 $\mathrm{c}$ 的稀释率分别选用 $20 \%$ 与 $10 \%$ ， 有效起到承上启下的作用; 最外一层耐磨层采用 $\mathrm{Co} 50+0.1 \% \mathrm{CeO}_{2}+10 \% \mathrm{Ni} / \mathrm{WC}$ 成分，稀释率控制在 $5 \%$ 以下, 采用较小的热输入, 避免 WC 颗粒过量熔 融降低最外层的耐磨性能并增加涂层开裂倾向。该 层设计厚度为 $1 \mathrm{~mm}$, 以确保在精磨加工后耐磨层 厚度在 $0.5 \mathrm{~mm}$ 以上。经工艺优化试验得到各层激 光增材制造工艺参数为: 连接层, 激光功率为 2000 $\mathrm{W}$ ，扫描速度为 $270 \mathrm{~mm} / \mathrm{min}$, 送粉率为 $3.24 \mathrm{~g} / \mathrm{min}$; 过渡层 $\mathrm{b}$ ，激光功率为 $1800 \mathrm{~W}$ ，扫描速度为 320 $\mathrm{mm} / \mathrm{min}$, 送粉率为 $5.62 \mathrm{~g} / \mathrm{min}$; 过渡层 $\mathrm{c}$, 激光功 率为 $1700 \mathrm{~W}$ ，扫描速度为 $300 \mathrm{~mm} / \mathrm{min}$, 送粉率为 $5.62 \mathrm{~g} / \mathrm{min}$; 耐磨层, 激光功率 $1900 \mathrm{~W}$ ，扫描速度 $350 \mathrm{~mm} / \mathrm{min}$, 送粉率 $7.65 \mathrm{~g} / \mathrm{min}$ 。各层工艺光斑直 径均为 $4 \mathrm{~mm}$ ，搭接率均采用 $50 \%$ 。

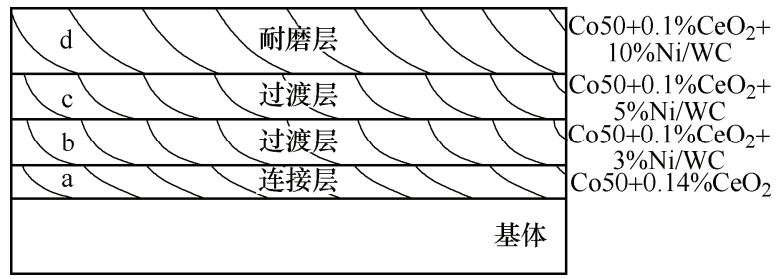

图 3 梯度涂层结构示意图

\section{3 凸轮边缘防塌陷夹具设计}

增材制造过程中，凸轮边缘在激光作用下与粉 末同时受热熔化, 熔融的金属液体会向凸轮侧壁下 方流动, 从而产生边缘塌陷现象, 影响后续加工与
成品使用性能 ${ }^{[18]}$ 。为解决这一问题, 在凸轮两侧分 别安装材质为纯铜的保护套，如图 4 所示。由于纯 铜在增材制造过程中不易与基体和粉末互溶，有效 限制了熔融金属液体的流动性，使其无法流向凸轮 侧壁，从而保护凸轮边缘形状完好。该方法简便易 行且保护效果良好。

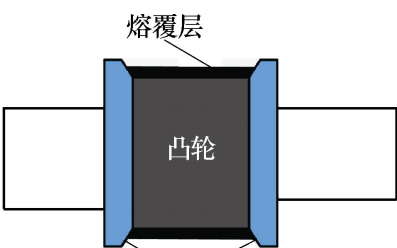

保护夹具 ( 纯铜)

(a) 凸轮边缘防塌陷夹具示意图

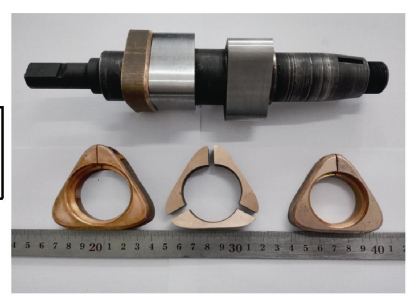

(b) 凸轮边缘防塌陷夹具实物图
图 4 凸轮轴边缘防塌陷夹具

\section{4 凸轮运动控制设计}

增材制造过程中激光束始终垂直凸轮上某点 的水平切线, 以此保证激光束法向入射凸轮表面。 如图 5 所示，在凸轮轮廓线上等角度插入一系列点 $A_{1}, A_{2}, \cdots, A_{n}$, 各点坐标为 $\left(x_{1}, z_{1}\right),\left(x_{2}, z_{2}\right), \cdots$, $\left(x_{n}, z_{n}\right)$ ，插入点的切线斜率为 $k_{1}, k_{2}, \cdots, k_{n}$, 通过 $A_{2}, A_{3}, \cdots, A_{n}$ 分别做该点法线 $\overline{A_{2} B_{2}}, \overline{A_{3} B_{3}}, \cdots$, $\overline{A_{n} B_{n}}$ ，再由 $O$ 点做垂直于 $\overline{A_{2} B_{2}}, \overline{A_{3} B_{3}}, \cdots, \overline{A_{n} B_{n}}$ 的直线 $\overline{O B_{2}}, \overline{O B_{3}}, \cdots, \overline{O B_{n}}$ 。

由于 $\overline{O B_{n}}$ 与 $A_{2}, A_{3}, \cdots, A_{n}$ 各点处的切线平 行, 故 $\overline{O B_{n}}$ 直线斜率同为 $k_{n}$ 。

当 $\overline{A_{2} B_{2}}, \overline{A_{3} B_{3}}, \cdots, \overline{A_{n} B_{n}}$ 处于竖直状态时, 激光束沿法线入射。此时, $\overline{A_{2} B_{2}}, \overline{A_{3} B_{3}}, \cdots, \overline{A_{n} B_{n}}$ 的长度增量为机床 $Z$ 轴的移动量 $\Delta z_{n}$

$$
\Delta z_{n}=\overline{A_{n} B_{n}}-\overline{A_{n-1} B_{n-1}}
$$

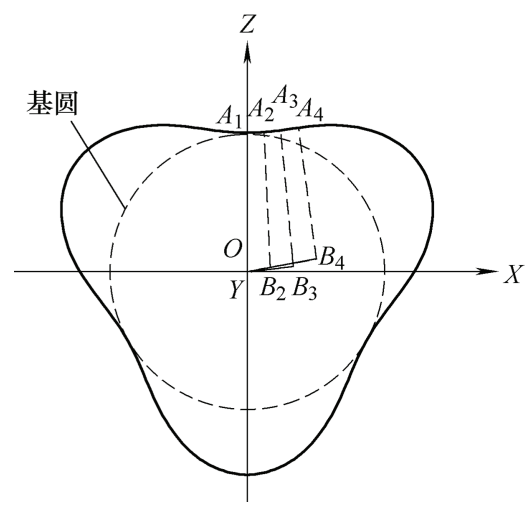

图 5 凸轮运动控制数学模型

$\overline{O B_{2}}, \overline{O B_{3}}, \cdots, \overline{O B_{n}}$ 的长度增量为机床 $X$ 轴 
的移动量 $\Delta x_{n}$

$$
\Delta x_{n}=\overline{O B_{n}}-\overline{O B_{n-1}}
$$

直线 $\overline{O B_{n}}$ 与直线 $\overline{O B_{n-1}}$ 的夹角为转台转动 量 $\Delta \omega_{n}$

$$
\Delta \omega_{n}=\arctan \left|k_{n}\right|-\arctan \left|k_{n-1}\right|
$$

式(1)中线段 $\overline{A_{n} B_{n}}$ 的长度为

$$
\overline{A_{n} B_{n}}=\frac{\left|z_{n}-k_{n} x_{n}\right|}{\sqrt{1+k_{n}^{2}}}
$$

式(2)中线段 $\overline{O B_{n}}$ 的长度为

$$
\overline{O B_{n}}=\frac{\left|x_{n}\right|+\left|k_{n}\right|\left|z_{n}-k_{n} x_{n}\right|}{\sqrt{1+k_{n}^{2}}}
$$

式(4)与式(5)中由 $\overline{O B_{n}}$ 函数式 $z=k_{n} x$ 可知 $k_{n} x_{n}$ 为 $\overline{O B_{n}}$ 的 $Z$ 轴坐标值。

凸轮每段圆弧进给速度 $F_{n}$ 为

$$
F_{n}=\sqrt{\left(\frac{\Delta x_{n}}{t}\right)^{2}+\left(\frac{\Delta z_{n}}{t}\right)^{2}}
$$

式(6)中激光束扫过每段圆弧的扫描时间 $t=S / V_{\mathrm{s}}$ ，每段圆弧长度 $S=\rho \pi / n$, 其中曲率半径 $\rho=R_{0}+h, n$ 为凸轮圆弧分段数， $R_{0}$ 为基圆半径， $h$ 为凸轮外轮廓测量点到基圆的距离。

对凸轮轮廓曲线每隔一度测量, 共测量 360 个 点, 部分数据如表 1 所示。

表 1 凸轮测量数据

\begin{tabular}{ccc}
\hline \multirow{2}{*}{ 测量点 } & \multicolumn{2}{c}{ 所测变量 } \\
\cline { 2 - 3 } & 角度 $\theta /\left(^{\circ}\right)$ & 距离 $h / \mathrm{mm}$ \\
\hline$A_{1}$ & 0 & 0.006 \\
$A_{2}$ & 1.00 & 0.024 \\
$A_{3}$ & 2.00 & 0.055 \\
$A_{4}$ & 3.00 & 0.097 \\
$A_{5}$ & 4.00 & 0.153 \\
$A_{6}$ & 5.00 & 0.221 \\
\hline
\end{tabular}

将极坐标数据 $(\rho, \theta)$ 导入 Matlab 中经过拟合 得出图 5 所示凸轮轮廓曲线。

图 6 为增材制造试验中凸轮运动控制流程图。 通过四轴联动激光数控加工机对凸轮轴的旋转及同 轴送粉头与数控工作台的移动实现协同控制，确保 扫描速度及送粉头与凸轮表面的距离不变, 从而实 现光斑直径、激光束入射方向及凸轮线速度始终不 变, 以获得厚度均匀的梯度涂层。

\section{5 结果分析}

5.1 凸轮轴激光增材制造

按前述梯度涂层设计方案与优化后的工艺参

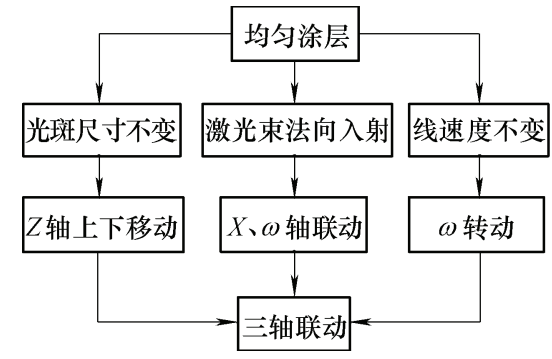

图 6 凸轮运动控制流程图

数，采用凸轮边缘防塌陷夹具并根据凸轮运动控制 方法在激光加工机上进行激光增材制造试验，如图 $7 \mathrm{a}$ 所示。图 $7 \mathrm{~b}$ 为激光增材制造后凸轮轴宏观形貌， 凸轮外形尺寸良好，梯度层表面光洁平整，便于后 续的精磨加工。将图 $7 \mathrm{~b}$ 所示凸轮轴表面精磨加工后 得到如图 7c 所示的凸轮轴成品件。如图 7d 所示， 梯度涂层均匀性良好，厚度约为 $2.5 \mathrm{~mm}$ 。

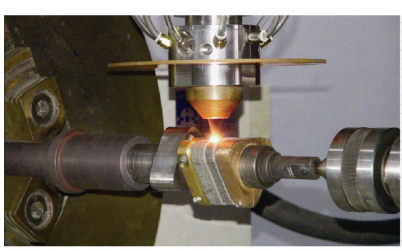

(a) 增材制造过程

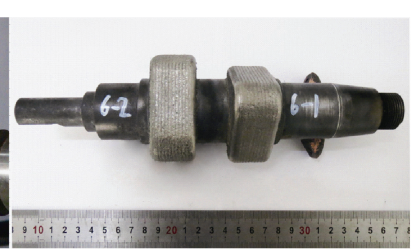

(b) 增材制造后

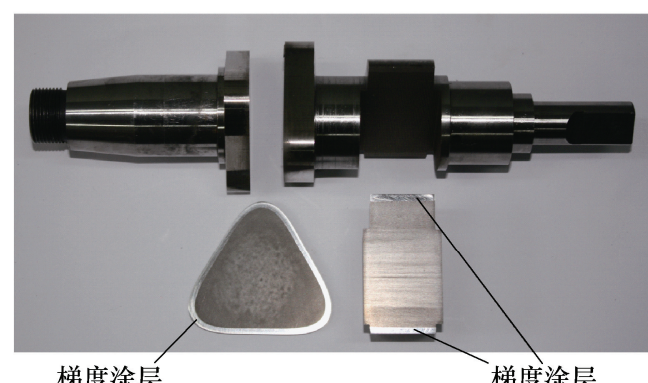

(c) 表面精磨后梯度涂层均匀性

图 7 增材制造试验过程与凸轮轴宏观形貌

\section{2 梯度涂层微观形貌分析}

图 8 为梯度涂层横截面形貌，由图 8 可见，梯 度层无气孔、裂纹产生。连接层与基体以及各梯度 层之间结合界面良好，其中过渡层与耐磨层中离散 分布着多棱角形态的 WC 颗粒。精磨加工后形成的 凸轮表面位于耐磨层中部，耐磨性能良好。

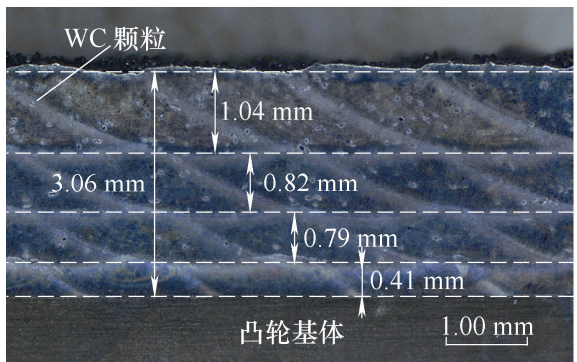

图 8 梯度涂层横截面形貌

图 9 为梯度涂层显微硬度分布。如图 9 所示, 
显微硬度由耐磨层表面至基体逐渐下降, 耐磨层平 均显微硬度达 $690 \mathrm{HV}$, 耐磨层表面向下 $0.6 \mathrm{~mm}$ 处 硬度约为 $720 \mathrm{HV}$ 。因此, 为保证磨削后耐磨层表面 具有较高硬度, 磨削量应控制在 $0.6 \mathrm{~mm}$ 左右。过 渡层与连接层由于 WC 质量分数逐渐减少以及回火 作用，硬度逐渐降低。

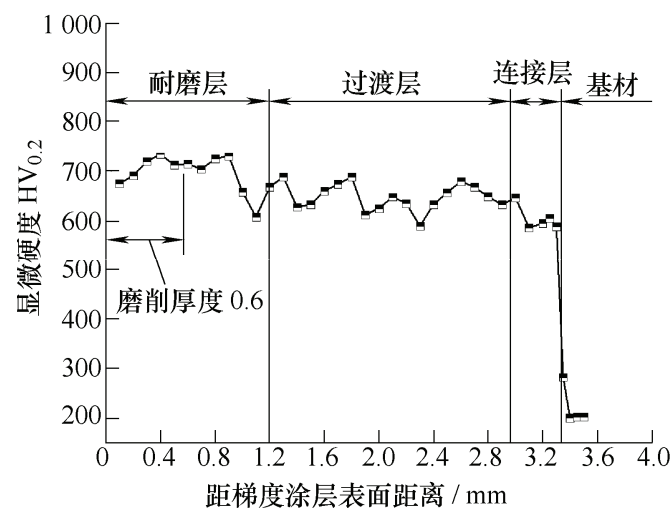

图 9 梯度涂层显微硬度分布曲线

图 10 为基体与连接层交界处微观组织形态。 连接层与基体结合界面处形成了一条宽约为 $4 \mu \mathrm{m}$ 的白亮窄带。连接层由下至上依次形成平面晶、柱 状晶与树枝晶。由于连接层与基体结合面位置的熔 池过冷度小、温度梯度 $G$ 较高且凝固速率 $R$ 较低, 在界面处形成平面晶结构。随着界面以上的温度梯 度 $G$ 减小与凝固速率 $R$ 增大, 柱状晶逐渐形成。随 着 $G / R$ 值继续减小, 熔体过冷度不断增大, 晶体结 构向树枝晶转变 ${ }^{[19]}$ 。

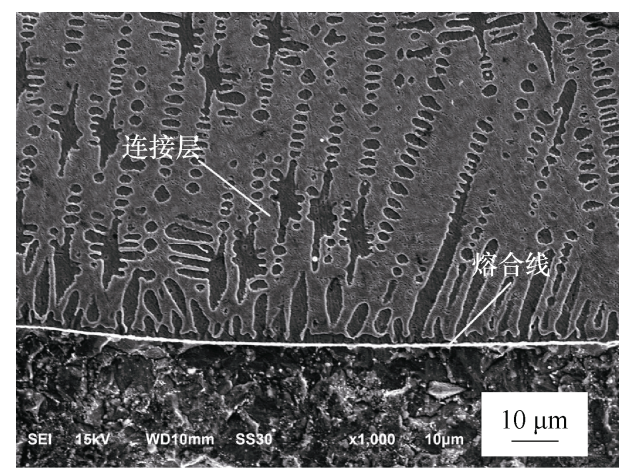

图 10 基体与连接层交界处微观形貌

图 11 为梯度涂层 $\mathrm{X}$ 射线衍射图谱。经标定发 现，连接层主要由 $\gamma-\mathrm{Co}$ 固溶体、 $\mathrm{Co}_{3} \mathrm{~B}$ 及 $\mathrm{M}_{23} \mathrm{C}_{6}$ 等 相组成。而过渡层与耐磨层除上述物相外, 还发现 了 $\mathrm{WC}$ 与 $\mathrm{W}_{2} \mathrm{C}$ 两相。两相在耐磨层中的衍射峰 强度明显高于过渡层, 说明耐磨层中 $\mathrm{WC}$ 与 $\mathrm{W}_{2} \mathrm{C}$ 的质量分数高于过渡层, 说明形成了良好的梯度过 渡。 $\mathrm{W}_{2} \mathrm{C}$ 主要是由 $\mathrm{WC}$ 部分分解产生, 两相的存在 提高涂层硬度的同时，会显著地提高表层的耐磨 性能。

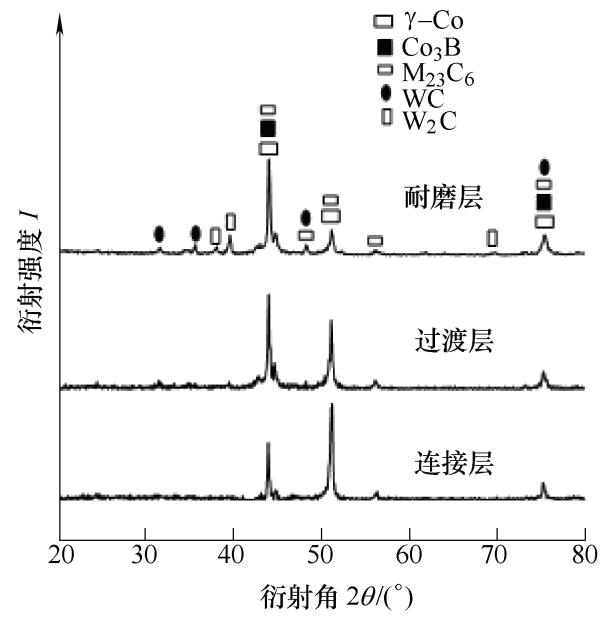

图 11 梯度涂层 XRD 衍射图谱

图 12 为梯度涂层扫描电镜微观组织形貌。各 梯度层由灰白色共晶与深灰色枝晶组织组成。连接 层 $\mathrm{a} 、$ 过渡层 $\mathrm{b}$ 和 $\mathrm{c}$ 与耐磨层 $\mathrm{d}$ 中灰白色共晶数量 依次减少。由于 $\mathrm{WC}$ 与 $\mathrm{CeO}_{2}$ 具有阻碍晶粒长大的 作用，梯度涂层的晶粒随各层 $\mathrm{WC}$ 与 $\mathrm{CeO}_{2}$ 含量的 增加而逐渐细化。增材过程中过渡层 $\mathrm{c}$ 对过渡层 $\mathrm{b}$ 产生回火作用，进而导致过渡层 $\mathrm{b}$ 出现晶粒粗化 现象。

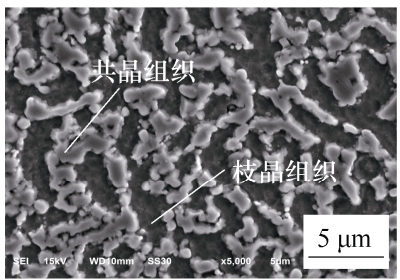

(a) 连接层

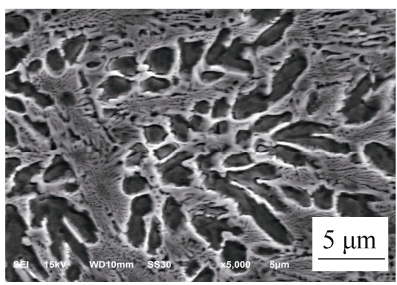

(c) 过渡层 $\mathrm{c}$

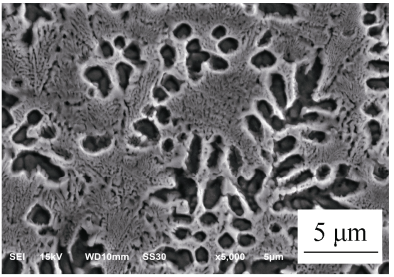

(b) 过渡层 b

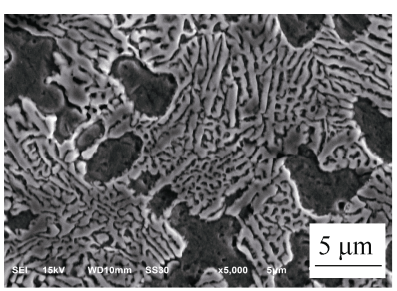

(d) 耐磨层
图 12 梯度涂层微观组织形貌

图 13 为梯度涂层中 WC 的 SEM 微观组织形貌 及 $\mathrm{W}$ 元素面扫描图谱。图 13a 为梯度涂层内部的 $\mathrm{WC}$ 颗粒以边缘部分扩散熔解的方式存在。由于梯 度涂层中 WC 在高温下只是部分边缘熔解, 其周围 形成细小的共晶组织, 这样既保证了 WC 颗粒在梯 度涂层内的结合强度, 又有效地起到了耐磨的作用, 同时又减小了裂纹开裂倾向。对图 13a 中 WC 颗粒 中心及周边位置的 $A 、 B 、 C$ 点进行 $\operatorname{EDS}$ 能谱检测， 其结果见表 2 。由表 2 所示的各元素原子分数可知， 图 13a 所示的颗粒为 WC, 边缘发生部分熔解, 近 
颗粒区域 $(B$ 点)除了 $\mathrm{WC}$, 开始出现 $\mathrm{Co}$ 的分布, 稍 远区域 $\left(C\right.$ 点)出现了 $\mathrm{W}_{2} \mathrm{C}$ 分布, 同时 $C o$ 的百分比 剧增。图 $13 \mathrm{~b}$ 为 $\mathrm{W}$ 元素 $\mathrm{EDS}$ 面扫描图, 进一步说 明 WC 颗粒在边缘熔解时 $\mathrm{W}$ 逐渐向涂层区域扩散 并与 Co 元素发生互溶。弥散分布的 WC 颗粒与 $\mathrm{WC} 、 \mathrm{~W}_{2} \mathrm{C}$ 增强相使梯度涂层的耐磨性得到显著 提高。

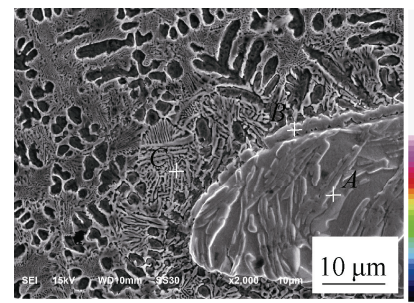

(a) WC 颗粒微观组织形貌

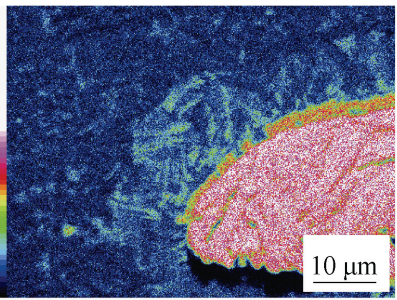

(b) W元素面扫描图谱
图 13 梯度涂层中 $\mathrm{WC}$ 的微观形貌及 $\mathrm{W}$ 元素面扫描图谱

表 2 WC 颗粒及其周围 EDS 成分分析结果(原子分数) \%

\begin{tabular}{cccccc}
\hline \multirow{2}{*}{ 扫描点 } & \multicolumn{5}{c}{ 元素种类 } \\
\cline { 2 - 6 } & $\mathrm{Cr}$ & $\mathrm{Ni}$ & $\mathrm{W}$ & $\mathrm{C}$ & $\mathrm{Co}$ \\
\hline$A$ & - & - & 44.15 & 55.85 & - \\
$B$ & 8.46 & - & 28.79 & 37.19 & 25.57 \\
$C$ & 31.49 & 3.16 & 19.38 & 9.02 & 36.94 \\
\hline
\end{tabular}

5.3 耐磨性能测试

在高压喷油洜试验台上对三对渗碳淬火与激 光增材制造凸轮轴进行使用寿命对比考核测试, 如 图 14 所示, 两种凸轮轴分别装在两台泉体中, 同周 期对比考核。

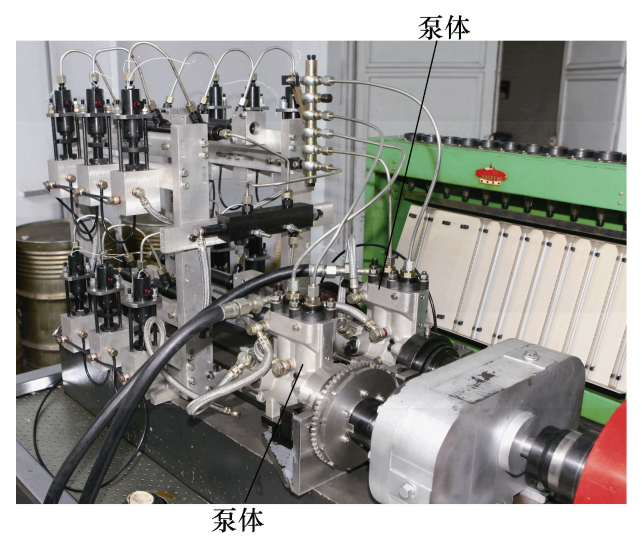

图 14 高压喷油厡试验台设备图

经台架试验，依据前述失效判据，渗碳淬火凸 轮轴平均使用寿命约为 $76 \mathrm{~h}$, 而激光增材制造凸轮 轴平均使用寿命约为 $126 \mathrm{~h}$ 激光增材制造凸轮轴较 渗碳淬火凸轮轴使用寿命提高约 $65 \%$ 。三对凸轮轴 的考核寿命如图 15 所示。表明激光增材制造梯度耐 磨层具有优异的耐磨性能与承受高载荷的能力。

图 16 为渗碳淬火与激光增材制造凸轮轴失效
后凸轮表面的磨痕形貌对比图片。由图 16 可见，渗 碳淬火与激光增材制造凸轮轴凸轮表面均出现条状 磨痕。其中渗碳淬火凸轮轴的凸轮表面磨痕较宽， 而激光增材制造的凸轮表面仅有少量窄而浅的磨 痕。表明激光增材制造后凸轮轴的使用寿命得到明 显提高。

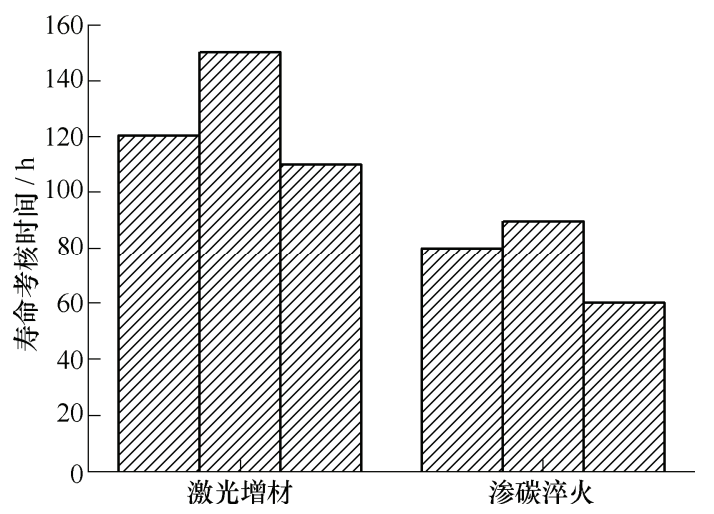

图 15 渗碳淬火与激光增材制造凸轮轴寿命考核时间

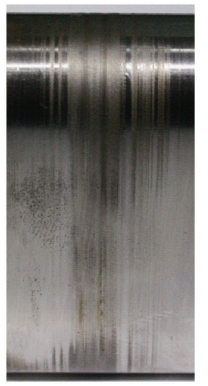

(a) 渗碳淬火凸轮轴

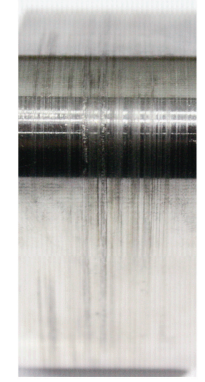

(b) 激光增材制造凸轮轴
图 16 渗碳淬火与增材制造凸轮轴失效后凸轮表面形貌

综上所述，通过多层激光沉积工艺控制与 $\mathrm{WC}$ 耐磨硬质颗粒的梯次分布，实现了激光沉积层显微 硬度的梯度分布，既保证了最外层具有优良的耐磨 性能、中间过渡层具有良好的承载与承接功能、底 层具有优异的连接性能, 同时又有效解决了高硬度 高厚度激光增材制造耐磨层裂纹产生难题。细密的 耐磨层微观组织与内嵌的边缘部分熔解 WC 硬质颗 粒, 为使表层具有优异耐磨性能提供了可靠与有效 的保障。大稀释率的底层激光沉积工艺, 使高硬度 的激光增材制造层与低硬度的母材可靠连接。这些 相互影响与关联的因素综合在一起，使激光增材制 造梯度耐磨层凸轮轴在后续的燃油喷射试验台可靠 性测试过程中，表现出优异的使用寿命。

\section{6 结论}

（1）通过采用梯度涂层结构、凸轮边缘防塌陷 夹具与凸轮运动控制方法实现凸轮表面梯度耐磨涂 
层的激光增材制造研究。梯度涂层成形良好、厚度 均匀且凸轮边缘未发生塌陷现象。

(2) 梯度涂层中主要由 $\gamma$-Co 固溶体、 $\mathrm{Co}_{3} \mathrm{~B}$ 及 $\mathrm{M}_{23} \mathrm{C}_{6}$ 等相组成。其中过渡层与耐磨层还出现 $\mathrm{WC}$ 与 $\mathrm{W}_{2} \mathrm{C}$ 相。微观组织主要表现为灰白色共晶组织与 深灰色枝晶组织。梯度涂层与基体形成良好的冶金 结合。

(3) 激光增材制造后的凸轮表面耐磨损性能明 显提高，使用寿命较渗碳淬火凸轮轴提高约 $65 \%$ 。

\section{参 考 文 献}

[1] 王大承. HT300 摩托车凸轮轴激光表面强化处理研究 $[\mathrm{J}]$. 激光技术，2002，26(6)：474-477.

WANG Dacheng. Research of laser surface strengthening technique for HT300 camshaft of motorcycle[J]. Laser Technology , 2002 , 26(6) : 474-477.

[2] 李双寿, 陆劲昆, 边庆月，等. 球墨铸铁凸轮轴的激光 表面熔凝处理 [J]. 金属热处理，2005，30(2)：4-8.

LI Shuangshou , LU Jinkun , BIAN Qingyue , et al. Laser surface melting process for camshaft made of ductile iron[J]. Heat Treatment of Metals , 2005 , 30(2) : 4-8.

[3] 卢求元, 赵俊平. 发动机凸轮表面耐磨减摩薄膜的制备 与表征[J]. 金属热处理, 2015，40(6)：171-174.

LU Qiuyuan , ZHAO Junping. Preparation and characterization of films with high wear resistance and low friction coefficient on engine cam surface[J]. Heat Treatment of Metals , 2015 , 40(6) : 171-174.

[4] RICCIARDI G , CANTELLO M. Surface treatment of automobile parts by $\mathrm{rtm}[\mathrm{J}]$. SPIE Vol.957, Laser Beam Surface Treating and Coating , 1988 : 66-74.

[5] 石岩, 张宏, 刘双宇, 等. 高硬度多微孔小型零件激光 表面精密熔覆钴基合金涂层提高耐冲蚀性能研究 $[\mathrm{J}]$. 机械工程学报，2011，47(2)：95-101.

SHI Yan ZZHANG Hong ,LIU Shuangyu ,et al. Improving resistance of cobalt-based alloy coating formed laser fine cladding on high-hardness and microporous small parts [J]. Journal of Mechanical Engineering ,2011,47(2) :95-101.

[6] RIABKINA-FISHMAN M ,RABKIN E ,LEVIN P, et al. Laser produced functionally graded tungsten carbide coatings on M2 high-speed tool steel[J]. Materials Science and Engineering , 2001 , 302(1) : 106-114.

[7] 张国栋, 杨辉, 王麒瑜, 等. 铁素体不锈钢激光熔覆层 组织和性能研究 [J]. 机械工程学报, 2016, 52(12)： $37-45$.

ZHANG Guodong, YANG Hui, WANG Qiyu, et al. Microstructure and properties of laser cladding layer on ferritic stainless steel[J]. Journal of Mechanical Engineering , 2016 , 52(12) : 37-45.

[8] 赵彦华, 孙杰, 李剑峰, 等. $\mathrm{KMN}$ 钢激光熔覆 $\mathrm{FeCr}$ 合 金修复层组织性能及耐磨、耐蚀性研究 [J]. 机械工程学 报，2015，51(8): 37-43.

ZHAO Yanhua ,SUN Jie ,LI Jianfeng, et al. Research on microstructure properties and wear and corrosion resistance of $\mathrm{FeCr}$ repaired coating on $\mathrm{kmn}$ steel by laser cladding[J]. Journal of Mechanical Engineering , 2015 , 51(8) : 37-43.

[9] 王新洪，张敏，邹增大，等. 激光熔覆 $\mathrm{TiC}_{\mathrm{p}} \mathrm{Ni}$ 基合金 复合涂层的显微组织与性能 $[\mathrm{J}]$. 机械工程学报，2003， 39(2) : 37-41.

WANG Xinhong, ZHANG Min, ZOU Zengda, et al. Microstructure and properties of laser clad $\mathrm{TiC}_{\mathrm{p}} / \mathrm{Ni}$-based alloys composite coating[J]. Chinese Journal of Mechanical Engineering , 2003 , 39(2) : 37-41.

[10] MAJUMDAR D J ,CHANDRA R B ,NATHB K A , et al. Studies on compositionally graded silicon carbide dispersedcomposite surface on mild steel developed by laser surface cladding $[\mathrm{J}]$. Journal of Materials Processing Technology , $2008,1: 505-512$.

[11] 沈斌, 间广超, 吴钢. 复杂盘形凸轮表面激光熔覆工艺 [J]. 中国激光，2009，36(1) : 244-248.

SHEN Bin , YAN Guangchao , WU Gang. Laser cladding on complex disc cam[J]. Chinese Journal of Laser , 2009 , 36(1) : 244-248.

[12] 董世运, 张晓东，徐滨士，等. 45 钢凸轮轴磨损凸轮的 激光熔覆再制造 $[\mathrm{J}]$. 装甲兵工程学院学报，2011， 25(2) : 85-87.

DONG Shiyun, ZHANG Xiaodong, XU Binshi, et al. Laser cladding remanufacturing of 45 steel camshaft worn cam[J]. Journal of Academy of Armored Force Engineering , 2011, 25(2) : 85-87.

[13] 宋建丽，邓琦林，陈畅源，等. 宽带激光熔覆高硬度火 焰喷涂层组织和裂纹行为 $[\mathrm{J}]$. 机械工程学报, 2006 , 42(12) : 169-174.

SONG Jianli, DENG Qilin, CHEN Changyuan, et al. Microstructure and cracking behavior of wide-band laser cladding flame sprayed high hardness coatings[J]. Chinese Journal of Mechanical Engineering , 2006 , 42(12) : 169-174.

[14] 余廷，邓琦林，董刚，等. 钽对激光熔覆镍基涂层的裂 纹敏感性及力学性能的影响 [J]. 机械工程学报, 2011 , 47 (22) : 25-30.

YU Ting , DENG Qilin , DONG Gang, et al. Influence of 
Ta on crack susceptibility and mechanical properties of laser clad ni-based coating[J]. Journal of Mechanical Engineering , 2011,47 (22) : 25-30.

[15] 陈列, 陶然, 刘顿, 等. 斜齿面激光熔覆的开裂倾向分 析及控制研究 $[\mathrm{J}]$. 中国激光，2014，41(11)：1-7.

CHEN Lie, TAO Ran, LIU Dun, et al. Analysis and control the cracking tendency on helical tooth surface during laser cladding[J]. Chinese Journal of Laser , 2014 , 41(11) : 1-7.

[16] WANG Dengzhi ,HU Qianwu ,ZENG Xiaoyan. Residual stress and cracking behaviors of $\mathrm{Cr} 13 \mathrm{Ni} 5 \mathrm{Si} 2$ based composite coatings prepared by laser-induction hybrid cladding[J]. Surface and Coatings Technology, 2015 , 274(6) : 51-59.

[17] 张栋栋. 高硬度激光熔覆层裂纹的产生机理及控制方 法研究[D]. 长春：长春理工大学, 2013.

ZHANG Dongdong. Study on the generating mechanism and control methods of high hardness laser cladding coating cracks[D]. Changchun : Changchun University of Science and Technology , 2013.

[18] 陈列, 谢沛霖. 齿面激光熔覆中的防边缘塌陷工艺研究 [J]. 激光技术，2007，31(5)：518-521.

CHEN Lie , XIE Peilin. Research of preventing the edges of tooth surface from collapsing in laser-cladding process[J]. Laser Technology , 2007 , 31(5) : 518-521.

[19] 袁庆龙, 冯旭东, 曹晶晶, 等. 激光熔覆镍基合金涂层 微观组织研究 [J]. 中国激光, 2010,37(8) : 2116-2120. YUAN Qinglong, FENG Xudong, CAO Jingiing, et al Research on microstructure of Ni-Based alloy coating by laser cladding[J]. Chinese Journal of Laser , 2010 , 37(8) : 2116-2120.

作者简介 : 石岩, 男, 1972 年出生, 博士 , 教授, 博士研究生导师。主 要研究方向为激光加工技术。

E-mail : lasercust@hotmail.com

李云峰(通信作者), 男, 1989 年出生, 博士研究生。主要研究方向为激 光加工技术。

E-mail : gyhu22@163.com

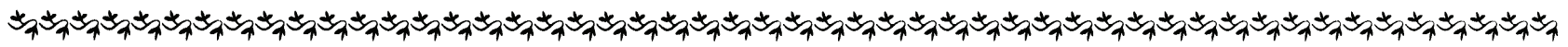

第 6 届上银优秀机械博士论文奖——佳作奖

\section{双机及多机驱动振动系统同步理论的研究}

作者：张学良

毕业学校 : 东北大学

指导教师 : 闻邦椿 赵春雨

在多原动机驱动偏心转子(简称激振器)的振动系统中, 常要求其能够实现同步运转, 为达这一目的, 以往多数采用刚性 传动, 如齿轮等进行强制同步, 其结果会使系统变得更为复杂, 并带来维修不便等诸多弊病。假如去掉如齿轮等强制同步装 置, 而仍然能够保证系统同步运转, 将会使机器结构变得更为简单紧凑。振动同步技术是解决这一问题的有效措施之一。目 前在许多工业部门，利用振动同步原理设计的自同步振动机械已经得到广泛应用，且创造出巨大的经济效益和社会效益。

本文针对两个及多个相同或不相同激振器在不同分布方式、不同旋转方向、不同振动条件(即超远共振和近共振)以及考 虑圆滚干摩擦的同步原理及机制, 做了一系列的理论、数值及试验研究。详细剖析了系统的耦合动力学特性; 设计出试验样 机, 完成振动测试 , 从而验证了理论结果的有效性及方法的可行性 ; 总结并提炼出解决振动同步问题所遵循的力学规则 广义动态对称性规则; 提出了双机及多机驱动同步理论的若干同步性判据与同步状态的稳定性判据; 最后将研究结果应用于 工程实际，建立了一套较为系统的振动同步理论体系与框架。主要创新点如下。

(1) 三个理论方面创新

1) 发展并完善了超远共振单质体多机驱动振动系统的同步理论。

2) 研究并提出了近共振双质体非线性振动系统的同步理论。

3) 研究并提出了振动系统中干摩擦条件下圆柱滚子的振动同步传动理论。

(2) 三个工程应用

1) 将超远共振振动系统相关同步理论应用到工程振动设备的设计中。

2) 将近共振振动系统同步理论应用到工程振动设备的设计中。

3) 将圆柱滚子的振动同步传动理论应用到新型振动破磨装备的研制开发中。

(3) 三个企业关键技术难题的解决

1) 对当前我国已设计制造出的世界最大振动篎 $\left(56 \mathrm{~m}^{2}\right)$ 进行工艺效果改善。

2) 对某企业超远共振自同步振动离心机进行理论指导及优化设计。

3) 对某企业两类近共振双质体非线性振动离心机进行改型优化设计。 